\title{
An Assessment of Overdose Surveillance at a Local Public Health Department
}

\author{
Samir T. Parmar, Brittany K. Yarnell \\ Epidemiology, Marion County Public Health Department, Indianapolis, Indiana, United States \\ Objective
}

To assess the data sources used to monitor overdose events in Marion County and improve community overdose surveillance.

\section{Introduction}

Mirroring public health response to infectious disease outbreaks, many public health departments are taking an outbreak management approach to respond to drug overdose surges [1-3]. The Marion County Public Health Department (MCPHD) has developed an overdose response plan (ORP) integrating drug overdose surveillance and community stakeholder response strategies. Effective drug overdose surveillance requires accurate and reliable data streams. This work assessed data sources utilized for county overdose surveillance and provided recommendations to improve overdose surveillance.

\section{Methods}

Data sources utilized as of September 2018 for opioid overdose surveillance in Marion County were assessed on utilization history by epidemiologists. General recommendations to improve overdose surveillance were created based on the findings. The three primary sources were emergency department data, ambulance run data, and death certificate data. Secondary sources included Indiana Poison Center (IPC) and toxicology data. General recommendations were generated based on challenges/solutions encountered and good practices observed from other health departments $[4,5]$.

\section{Results}

The assessment of data sources and utilization showed variation of data entry at the hospital level, limited identifiers in some cases, and varying timeliness ranges which may limit combined use of many data sources. The emergency department data source showed particular variation in data entry, limited unique identifier information), and no incident location information which impedes geographical surveillance. Periodic data checks by the ambulance service data holder appears to drastically increase data quality. Intermittent data feed drops from specific emergency departments also interfered with effective surveillance.

Recommendations were generated based on lessons learned during successful partnerships with Indianapolis Emergency Medical Services, IPC, and emergency departments and challenges encountered during overdose surveillance work (Figure 1).

In application of the strategy, the MCPHD is interested in linking data and looking for other ways to improve our overdose response to get a fuller picture of what is happening with overdoses, so we applied the steps in figure one to find areas of improvement. We found that limited identifiers and incomplete fields are our biggest challenge to linking datasets, so to combat these gaps we identified sources that have the necessary fields of interest and have been working with others to improve the data quality. Additionally, data sources will be evaluated on experiences with three categories: completeness in data fields, timeliness of data delivery, and consistency of data feed. Data quality measures were developed for completeness by fields present per record, timeliness by lag time from time added to time of event, and consistency by record counts per facility over time. We also recognized that meeting with partners is necessary to share how we are using the data and additional datasets that we might use in the future. Additionally we have been meeting with academic researchers so that we can expand our analyses to identify other issues related to overdoses. Finally, in order to make a difference in Marion County we are applying our findings to our outreach and interventions to hopefully prevent more overdoses and deaths.

Future plans include data partnerships include police drug arrest data, fire department naloxone administration data, prescription drug monitoring data, Medicaid claims data, and health information exchange overdose data. Future research partnerships will consider a solutions based framework [6].

SDS Annual Conference Proceedings 2019. This is an Open Access article distributed under the terms of the Creative Commons AttributionNoncommercial 4.0 Unported License (http://creativecommons.org/licenses/by-nc/3.0/), permitting all non-commercial use, distribution, and reproduction in any medium, provided the original work is properly cited. 


\section{Conclusions}

The results of our work demonstrate the value in surveillance assessment to summarize limitations of the many data sources utilized at a local level to conduct overdose surveillance. Our evaluation approach provides a path to improve and fill in surveillance gaps with new processes. Other health departments interested in optimizing overdose surveillance may seek a similar evaluation approach. Periodic data linkages have not been implemented which presents an opportunity to glean valuable insights on longitudinal patterns of drug use in the population. Future collaboration with researchers presents an opportunity to improve MCPHD ORP, Safe Syringe Access and Support Program, and Substance Use Outreach Services interventions.

\section{Acknowledgement}

Thanks to Tammie Nelson and Joe Gibson of the Marion County Public Health Department for supporting this endeavor and assisting with writing.

\section{References}

1. Moore K, Boulet M, Lew J, Papadomanolakis-Pakis N. 2017. A public health outbreak management framework applied to surges in opioid overdoses. J Opioid Manag. 13(5), 273-81. PubMed https://doi.org/10.5055/jom.2017.0396

2. Rudd RA. 2016. Increases in drug and opioid-involved overdose deaths-United States, 2010-2015. MMWR Morb Mortal Wkly Rep. 65. PubMed https://doi.org/10.15585/mmwr.mm655051e1

3. Rowe C, Wheeler E, Jones TS, Yeh C, Coffin PO. 2019. Community-Based Response to Fentanyl Overdose Outbreak, San Francisco, 2015. J Urban Health. 96(1), 6-11. PubMed https://doi.org/10.1007/s11524-018-0250-x

4. Chen H, Hailey D, Wang N, Yu P. 2014. A review of data quality assessment methods for public health information systems. Int J Environ Res Public Health. 11(5), 5170-207. PubMed https://doi.org/10.3390/ijerph110505170

5. Massachusetts. Department of Public Health. An Assessment of Opioid-Related Deaths in Massachusetts (2013-2014). Massachusetts Department of Public Health; 2016.

6. Wiehe SE, Rosenman MB, Chartash D, Lipscomb ER, Nelson TL, et al. 2018. A Solutions-Based Approach to Building Data- Sharing Partnerships. EGEMS (Wash DC). 6(1). PubMed https://doi.org/10.5334/egems.236

Figure 1
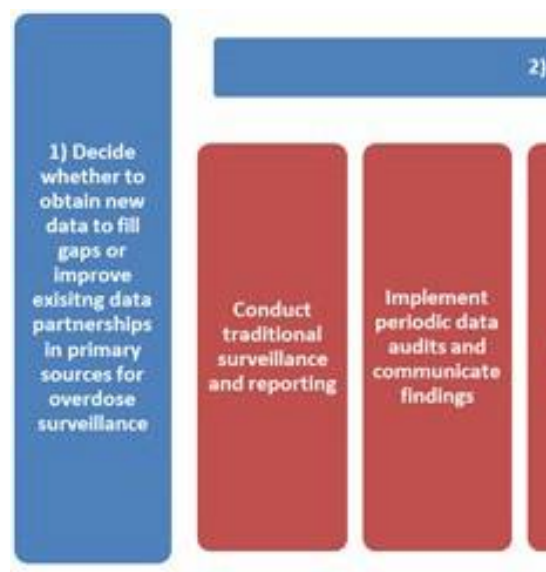

\section{2) Use the data:}
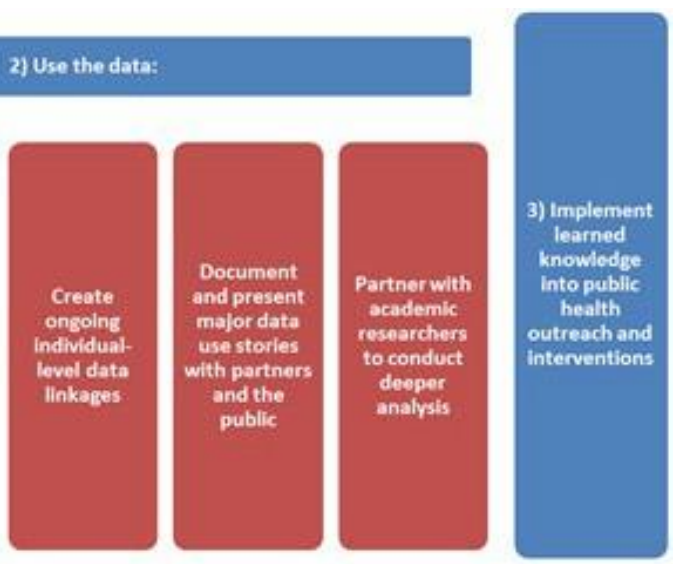

ISDS Annual Conference Proceedings 2019. This is an Open Access article distributed under the terms of the Creative Commons AttributionNoncommercial 4.0 Unported License (http://creativecommons.org/licenses/by-nc/3.0/), permitting all non-commercial use, distribution, and reproduction in any medium, provided the original work is properly cited. 